\title{
MedienPädagogik
}

Zeitschrift für Theorie und Praxis der Medienbildung

Themenheft Nr. 30: Medienpädagogik und Erwachsenenbildung. Herausgegeben von Bernhard Schmidt-Hertha und Matthias Rohs.

\section{Berufsbezogenes Lernen mit Twitter und YouTube: Social Media als amorpher Raum für Vernetzung und für die Bildung von Lerngemeinschaften}

Joanna Burchert und Michael Burchert

\begin{abstract}
Zusammenfassung
Berufliche Weiterbildung findet jetzt auch onlinegestützt statt. Oft bleibt allerdings offen, wie der pädagogische Nutzen digitaler Medien für berufsbezogenes Lernen einzuschätzen sei. Als Vorteil von Lernumgebungen wird z.B. gesehen, dass Lernenden Ressourcen so bereitgestellt werden können, dass diese unabhängig von zeitlichen und räumlichen Einschränkungen verfügbar sind. Andererseits steht in Frage, ob durch solche Zurverfügungstellung von Lernmaterialien auch Prozesse der Sinnstiftung und Reflexion, der persönlichen Weiterentwicklung unterstützt werden. In diesem Artikel spüren wir dieser pädagogischen Frage nach und zeigen anhand von zwei Beispielen aus informellen Lernzusammenhängen - Twitter und YouTube - wie berufsbezogenes Lernen mit digitalen Medien schon erfolgt. Wir reflektieren diese empirisch vorgefundenen Lernformen anhand der Netzwerk-Metapher, mit Bezugnahme zur Theorie situierten Lernens (Resnick et al. 1997, Lave/Wenger 1999, Fox 2000) und des Knowledge Building-Ansatzes (Scardamalia/ Bereiter 2014). Aus diesen Überlegungen resultieren Anregungen zum Nachdenken über die Medienkompetenz von Fachkräften und zu digital gestützten formellen Angeboten der beruflichen Weiterbildung. Wir plädieren für die Überschreitung von Grenzen formeller Lernzusammenhänge und die kritisch-reflexive Nutzung der schon digital bestehenden, amorphen Lernräume, um das berufsbezogene Lernen sinnvoller und fruchtbarer mitzugestalten. Das schliesst einen breit gefächerten Medienkompetenzbegriff ein.
\end{abstract}

Work-related learning with Twitter and YouTube: Social Media as amorphous space for networks and the constitution of learning communities

\begin{abstract}
Continuing vocational education and training is now also taking place online. Often, however, the pedagogical value of digital media for job-related learning remains unclear: on the one hand, there is the chance of independent use of learning resources; on the other hand, it is questionable whether and how digital tools can support processes of meaning making and reflection. In this article, we look at this educational question and, using two examples from informal learning contexts - Twitter and YouTube - describe why and how digital media here supports job-related learning. We reflect these empirically found
\end{abstract}


learning forms with reference to the network metaphor, the theory of situated cognition (Resnick et al. 1997, Lave/Wenger 1999, Fox 2000) and the knowledge building approach (Scardamalia/Bereiter 2014). These considerations lead to broader, critical-reflective media literacy concept for vocational education of specialists. We want to empower craftsmen and professionals to cross the boundaries of formal training, to participate in social media interest groups and to shape amorphous vocational education settings.

\section{Berufliche Weiterbildung und digitale Medien ${ }^{1}$}

"Kinder wachsen in eine Welt hinein, die von den Erwachsenen gestaltet ist. Sie müssen sich auf die geltenden Regeln einlassen, grundlegende Kulturtechniken aneignen, die Welt so aneignen, wie sie ist. Erwachsene verfügen über diese Kulturtechniken, sie sind diejenigen, welche die Welt gestalten. Erwachsene verfügen über ein grosses Wissen im Beruf und im Privaten, haben zu allen Fragen ihrer Person und ihres gesellschaftlichen Umfeldes Kenntnisse und vor allem eine eigene Meinung und Interpretation, haben eine persönliche und berufliche Identität. Alles das, was Erwachsene neu lernen, bedeutet gleichermassen ein Verlernen bereits vorhandenen Wissens, vor allem aber ein Uminterpretieren von Kenntnissen, eine Arbeit an der eigenen Identität. Erwachsene lernen interessengeleitet, von ihren eigenen Erfahrungen ausgehend, ordnen alles Neue in schon bestehende kognitive und subjektive Wahrnehmungen ein. Das Lehren von Erwachsenen muss berücksichtigen, dass Erwachsene bereits ihr Leben meistern, selbstbewusst und kompetent sind» (von Rein 2000).

Ein grosser Teil des Lernens erwerbstätiger Erwachsener ist berufsbezogen und eingebettet in den Arbeitsplatz - angefangen bei der Orientierung im Umgang mit neuen Aufgaben oder Werkzeugen über das Beheben von Problemen, Innovieren von Arbeitsprozessen, die kontinuierliche Erweiterung des eigenen Wissens- und Fertigkeitsstandes, die Befriedigung von Neugierde, die Vorbereitung von Karrieren oder die Vermeidung von Entlassungsgründen (vgl. Kade/Seitter 1996, Burchert et al. i.V.).

Digitale Medien werden seit Langem zur Ergänzung von Bildungsprozessen vorgeschlagen - und das, obwohl im allgemeinbildenden Bereich bestenfalls milde positive Effekte auf das Lernen und die Motivation belegt sind (z.B. Hattie 2009, Beiträge auf www.nosignificantdifference.org). Auch in der beruflichen Weiterbildung gibt es Bestrebungen, verstärkt digitale Medien zur Unterstützung von Lernprozessen einzusetzen (z.B. Bundesverband Betriebliche Weiterbildung 2016). Ein wichtiges Argument hierfür ist die Kostensenkung: durch die Reduzierung von Honoraren

1 Der vorliegende Artikel entstand durch die Anregung aus und unter Nutzung von Erkenntnissen aus drei laufenden Forschungsprojekten: dem BMUB- und ESF-geförderten Vorhaben nabus (https://weiterbildung.nznb.de/); dem BMBF-Projekt DiEDa (http://www.lernen-neu-denken.de); dem ebenfalls durch das BMBF geförderten Verbundprojekt lasidig (http://projekt-lasidig.info/ ). 
für Weiterbildende und den Wegfall von Anreise- und Übernachtungskosten werde das Training erschwinglicher - und dadurch vielleicht auch mehr Arbeitnehmenden zugänglich. Zudem ermögliche digital gestütztes Lernen die Nähe zu Arbeitsprozessen, so dass der Transfer des Gelernten wahrscheinlicher sei. Einschätzungen zur Lernförderlichkeit liegen hier bisher kaum vor: Wie immer, wenn es um Medien und Methoden geht, ist letztendlich nicht das einzelne Tool, sondern das didaktische Setting, in das es eingebettet ist, entscheidend für seine Wirksamkeit. Die meisten Angebote beruflicher Weiterbildung wirken in ihrer Nutzung digitaler Medien eher konservativ: es werden v.a. Lernressourcen (Texte, Videos, Quizze) auf Plattformen bereitgestellt. Diese Zurückhaltung mag damit zu erklären sein, dass eine Vielzahl von Herausforderungen bei der institutionellen Implementierung digitaler Medien zu beachten ist (Burchert/Grobe 2017). Auch scheint didaktisch nicht geklärt, worin der Mehrwert einer digitalen Lernanwendung für berufsbezogenes Lernen liegen kann: der Chance auf Bereitstellung und breite Verteilung von Lernressourcen steht z.B. die Ratlosigkeit gegenüber, wie Prozesse der Sinnstiftung und Reflexion, wie Erfahrungen digital vermittelbar sind. Der Frage nach dem pädagogischen Sinn digitaler Lernsettings möchten wir in diesem Artikel nachgehen, indem wir bestehende Praktiken berufsbezogenen Lernens im Internet betrachten und mit Bezug zu der Theorie situierter Kognition reflektieren. Wir möchten damit einerseits den Blick auf gelingende berufsbezogene Lernpraktiken online lenken, andererseits zum Nachdenken über Medienkompetenzförderung und digitale Medien in der formalen beruflichen Weiterbildung anregen.

Als Anwendungsfälle wählten wir die social media-Kanäle YouTube und Twitter. Der erste Dienst fokussiert Videos; der zweite ist durch die Darstellung kurzer Texte, Bilder und Grafiken, vor allem aber durch Verlinkung zu anderen Quellen charakterisiert. YouTube und Twitter zählen einer vielzitierten Umfrage von Hart (2016) zufolge zu den beliebtesten digitalen Werkzeugen zum Lernen - auch für die berufliche Weiterbildung. Bisher ist wenig darüber bekannt, wie hier berufsbezogenes Lernen durch die Akteure strukturiert wird, woher sie z.B. die Motivation beziehen, aktiv Wissen zu teilen oder ihre Kompetenzen weiterzuentwickeln. Auch das Verhältnis zwischen digital gestützter und analoger Kommunikation ist für Beziehungen, die in Social Communities entstanden, nicht geklärt. Und schliesslich weist Podrath (2016) darauf hin, dass Social Media wie YouTube eine «Schlüsselressource für jede Art von Weiterbildung» sei und ruft Weiterbildungsverantwortliche dazu auf, Hilfestellungen für Lernende im Umgang mit der solchen Plattformen zu entwickeln. Wie aber sieht Lernen bei Twitter und YouTube überhaupt aus? Im Folgenden beschreiben wir dazu Beispiele aus einem Interview und aus Dokumentenanalysen. 
Beispiele für berufsbezogenes Lernen mit digitalen Medien

\section{Vernetzen mit Twitter}

Der Nachrichtendienst Twitter wird oft als Micro-Blogging beschrieben: beschränkt auf 280 Zeichen [Stand 2018] können Informationen geteilt werden; oft werden Bilder oder animierte Grafiken, Hasthags als thematische Verknüpfungen und/oder Links in die Nachricht eingefügt. Im Folgenden beschreiben wir unter Bezugnahme auf ein Interview, wie eine Lehrerin Twitter nutzt, um berufsbezogen zu lernen.

Lisa ist seit 2009 bei Twitter aktiv:

"Ich glaub ich habe den [Twitter-]Account eingerichtet, als ich damals meine Webseite hab machen lassen [...] und dann auf Xing unterwegs war, bzw. später habe ich mich dann angemeldet bei LinkedIn und Google und dann gehörte es irgendwie mit dazu, auch auf Twitter zu sein.» (Z. 28 ff.)

Lisa beschreibt ihre Twitter-Aktivitäten als zunächst passiv, also «erstmal nur gelesen, also geguckt: wem folge ich» (Z. 33f.). Sie trifft die Auswahl an Personen, welchen sie folgt, anhand von für sie interessanten Tweets und Verlinkungen auf Blogs, Artikel etc.:

"Ein wichtiges Element in diesen Tweet sind ja die Verlinkungen; also man sucht sich Leute aus, die etwas twittern [...] - Leute, die verlinken auf interessante Artikel, weil man bei den 140 Zeichen ja begrenzt ist [...] Also das heisst man sieht jede Menge Links auf Artikel, die einen interessieren und sucht sich da halt sein Gebiet aus.» (Z. 34 ff.)

Über diese Gebiete definieren Twitter-Nutzerinnen und -nutzer ihre Interessen und verlinken sich mit Gleichgesinnten. Das Finden und Gefunden-Werden stehen dabei an zentraler Stelle (Z. 55 f.). Die so entstehenden Netzwerke verlaufen über Berufsgruppen-, Länder- und Kontinentalgrenzen hinweg.

2013 lernt Lisa bei einer Tagung den Twitter-Chat \#EDchatDE² kennen. Die wöchentlichen, etwa eine Stunde dauernden Treffen für Bildungsakteurinnen und -akteure auf Twitter sind für Lisa eine Weiterbildungsplattform für die Themenfelder Digitale Medien und Schule:

«Also jedenfalls wenn die Leute etwas machen, wie sie sozusagen Bildung mit digitalen Medien bzw. heutzutage halt darstellen möchten, dann bloggen sie in der Regel darüber, schreiben etwas, und das findet man halt dort» (Z. 159 ff.)

«Der André Spang der hat zusätzlich noch ein Wiki eingerichtet, [...] da werden quasi jede Woche die Tweets eingesammelt, die innerhalb dieser einen Stunde geschrieben wurden- zu diesem speziellen Thema -, so dass dieses Wiki jetzt

2 Siehe auch Larbig und Spang (2017) sowie https://edchatde.wordpress.com, http://www.lernen-neu-denken.de/2017/04/07/berufliche-weiterbildung-2-0-fuer-paedagoginnen-edchatde/. 
ein tolles Nachschlagewerk ist, wenn man wissen möchte zu einem bestimmten Thema, was wurde denn da geschrieben. Wöchentlich treffen sich etwa das variiert so ein bisschen oder normalerweise $50-\mathrm{hm}, 100$ hatten wir auch schon mal, soviel sind es nicht, vielleicht sind es auch manchmal nur 40 , aber jedenfalls werden quasi in dieser Stunde, ich sag mal, 500 bis 1000 Tweets geschrieben.» (Z. 78 ff.)

Der Umgang mit Twitter muss im Rahmen eines solchen Lernformates kompetent sein: Lisa verweist hier auch auf die Nutzung des Übersichtstools TweetDeck.

Die Bildungsakteurinnen und -akteure, mit welchen Lisa in Kontakt steht, nutzen Web 2.0 Präsenzen wie Twitter, Wiki und Wordpress um sich auszutauschen, zu verlinken und zu lernen. Der Kontakt bleibt jedoch nicht virtuell:

«So, und jetzt ist halt dieses Spannende, dass man sozusagen nicht einer anonymen Masse gegenübersteht sondern inzwischen, sag ich mal, aufgrund der verschiedenen Real Life Treffen auf EDUCamps oder BarCamps oder Konferenzen oder Kongressen - kenn ich von diesen, ich sag mal 100 Leuten, inzwischen auch schon ja 40 bestimmt persönlich.» (Z. 106 ff.)

Das Netzwerken entwickelt sich zum zentralen Element in Lisas Twitter- und Web 2.0 Nutzung: Sie folgt den Leuten, die sie interessieren, online und spricht sie gezielt an, wenn sie persönlich auf sie trifft (z.B. Z. 115 ff.). Dadurch verstärkt sich der digitale Austausch nochmals:

«Oder aber: wenn die Leute auf verschiedenen Konferenzen ihr Thema vorgestellt haben, dann sagen sie: ich schreibe jetzt dazu einen Blog-Artikel und den kann man dann halt nachlesen.» (Z. 140 ff.)

«Das hat sich entwickelt, dass man jetzt ganz viele Leute persönlich kennt und wenn ich jetzt sehe, da schreibt Frau X oder Herr Y oder wie auch immer, dann reagiere ich natürlich schneller.» (Z. 274 ff.)

"Also was ich das Faszinierende finde ist, dass ich nicht nur Infos bekomme, sondern dass ja diese Community entstanden ist, also diese Vernetzung [...] also was ich wirklich faszinierend finde, also ich duze im Prinzip alle mit denen ich hier da auf dem EDchatDE unterwegs bin.» (Z. 280 ff.)

Das Duzen ist eine Kommunikationsstrategie, die nicht nur im \#EDchatDE, sondern auch z.B. bei EduCamps eingesetzt wird, um Austausch zu erleichtern. Auch im Interview nennt Lisa Personen aus ihrem Umfeld beim Vornamen und erzählt beispielsweise ausführlich und begeistert von einem Treffen mit einer Bundestagsabgeordneten und einem ausländischen Kollegen, zu welchen sie über den Twitter-Chat den Kontakt herstellte.

Lisa beschreibt den \#EDchatDE als ihre «wöchentliche Fortbildung» (Z. 235). Daneben vernetzt sie sich selbstorganisiert v.a. über Twitter. Sie resümiert: 
"Also ich finde Weiterbildung muss, damit sie effektiv wird, die Möglichkeit bieten, dass ich sagen kann: hmm passt irgendwie zu mir und meinem Alltag, meiner Aufgabe, wenn ich eine Frage habe, ich weiss an wen ich mich wenden kann, geht ganz schnell, was weiss ich, wenn ich mir hier auf Twitter gegenseitig folge, kann ich mir Nachrichten schicken.» (Z. 713 ff.)

«Es sind halt viele dieser Lehrkräfte halt auch fleissig am Bloggen, so dass man dann im Prinzip nachlesen kann. Also ich habe zum Beispiel M gefragt: ich würde gerne in der Schule GoogleDrive anwenden, hab aber das Problem von wegen, ich soll ja keine personenbezogenen Daten verwenden, also sprich die E-Mail-Adresse der Schüler. Hat sie gesagt: ach, guck, ich hab des dazu geschrieben auf meinem Blog, du kannst es [ein Google Drive Dokument] auch einfach so frei geben, nur der Link ja und dann kann jeder, der den Link kennt, da mitschreiben.» (Z. $127 \mathrm{ff}$.)

In diesem Beispiel ist berufsbezogenes Lernen eine aktive Vernetzung von Expertinnen und Experten, die zur Beantwortung von Fragen aus dem Berufsalltag beiträgt und diesen inspiriert. Im Rahmen des \#EDchatDE werden gemeinsame Themen diskutiert, als Beiträge in einem Wiki zusammengetragen und auch Interessierten ausserhalb dieser Lerngruppe zur Verfügung gestellt. Der \#EDchatDE ist als strukturierte Kommunikationsform dem Austausch förderlich, aber aufgrund seiner zeitlichen und jeweils inhaltlichen Begrenzung nicht hinreichend um zu erklären, wie das berufsbezogene Lernen mit Twitter funktioniert. Dieses Lernen durch Vernetzung hat amorphen Charakter, d.h. um an dem Lernprozess teilzuhaben, müssen die Twitternden direkt miteinander verbunden sein. Dieser amorphe Charakter impliziert zugleich Zufälligkeit, das Fehlen klarer Muster.

\section{Creative commons of practice auf YouTube}

Gertrud Wolf (2016) resümiert über die Interaktion bei YouTube:

"YouTuber unterscheiden sich von herkömmlichen Lehrpersonen dadurch, dass sie nicht mit dem Anspruch antreten, anderen etwas beizubringen, sondern das, was sie können, zu kommunizieren. [...] Sie sind selbst begeistert von der Sache, über die sie reden und wollen andere ebenfalls dafür begeistern». YouTube kann unverbindlich, als eine Art Bibliothek, verwendet werden. So erwähnten die Befragten in der Studie von Hart (2016), dass sie die Vielseitigkeit von YouTube in Hinblick auf Thema und Komplexität schätzen und dass sie die Möglichkeit nutzten, Aussagen von Expertinnen und Experten und Praxisbeispiele im Lehrzusammenhang zu zeigen.

YouTube kann aber auch zu einem sozialen Netzwerk werden, und hier wiederum die Herausbildung von Lernnetzwerken im Sinne von «creative commons of practice» 
unterstützen. In solchen Lernnetzwerken verschwimmen Grenzen zwischen Lernenden und Lehrenden ebenso wie disziplinäre Einschränkungen, wie sie typischerweise in analogen beruflichen Praxisgemeinschaften vorzufinden sind. Ein Beispiel dafür bilden YouTuber, die grenzüberschreitend mit Holz, Metall und Technik experimentieren wie Jimmy Diresta, John Heisz und Matthias Wandel. Zu ihrer Motivation, einen YouTube-Kanal zu betreiben, schreiben sie:

«I' $m$ a carpenter by trade and have worked in commercial construction since 1985. [...] I can't and won't cover (or follow) every safety rule when doing a project, and it's not my responsibility to train the people that watch my videos. [...] My videos are not comprehensive tutorials - they are a look at how I did the project and should be viewed as entertainment or a source of inspiration.» (John Heisz, https://www.youtube.com/user/jpheisz/about)

"Videos about woodworking, taking more of an engineering perspective on things. This channels started out as a place to have videos to go with the articles on my website (http://woodgears.ca). However, since then, the videos have taken on a life of their own.» (Matthias Wandel, https://www.youtube. com/user/Matthiaswandel/about)

«I make stuff for a living, what you see me do here is my Job. I have been using tools for over 40 years. I have developed my comfort level with tools through years of experience. DON'T DO THE DANGEROUS THINGS I DO. Thank You for watching and subscribing!» (Jimmy Diresta, https://www.youtube.com/user/ jimmydiresta/about)

Jeder der zitierten Videoblogger bringt seine berufliche Erfahrung in die Beiträge ein - dennoch stehen nicht berufliche Aufgaben im Fokus der Videos, sondern die Anwendung (über)fachlichen Könnens und das Experimentieren. Zwei der betrachteten Kurzdarstellungen distanzieren sich explizit von der Vorstellung, in den Videos grundlegende handwerkliche Kenntnisse oder Sicherheitsrichtlinien zu vermitteln damit stellen sie sich z.B. deutlich in Gegensatz zu Ausbilderinnen und Ausbildern (vgl. Burchert 2014). Mit diesen Hinweisen und dem Bezug auf Inspiration wird deutlich, dass die Filmemacher sich nicht als Lehrende sehen - eher als Begeisterer im Sinne von Getrud Wolf (s.o.). Zwei der genannten YouTuber haben über eine Million Follower bzw. Abonnentinnen und Abonnenten; jedes der von ihnen publizierten Videos wird lebhaft kommentiert. Unklar bleibt, welche ihrer Zuschauerinnen und Zuschauer ein berufliches Interesse haben und welche das Gesehene privat interessant finden: In den Kommentaren finden sich sowohl affirmative (oft allgemein bleibende), als auch kritische Kommentare und Detail-Nachfragen, aber selten ein Bezug zum Hintergrund der Rezipienten. 
In ihren Videos versuchen die YouTuber z.B. technische Systeme begreifbar zu machen und Alternativen zu bisherigen Macharten zu durchdenken. Deutlich ist, dass die YouTuber untereinander in Verbindung stehen: so tauchen selbst hergestellte Werkzeuge des einen in der Werkstatt anderer Macherinnen und Macher auf - sei es als Geschenk, sei es als eigene Interpretation einer Idee. Ein Beispiel dafür sind Push Sticks, die bei Arbeiten mit Kreissägen genutzt werden, um Schnittverletzungen zu vermeiden. Das Design der Push Sticks wird untereinander aufgegriffen und individuell variiert. Abbildung 1 zeigt als Beispiel einen Screenshot mit Bezugnahme zu einem Push Stick von John Heisz, einem von Matthias Wandel und einem vergleichenden Video zweier weiterer YouTuber.

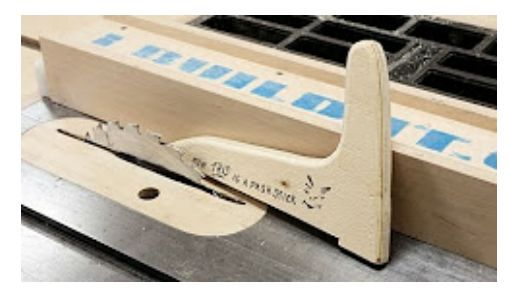

How To Make And Use The Worlds Best Push

Stick

I Build It

1 year ago $\cdot 94,677$ views

Website article and template here:

http://www.ibuildit.ca/Workshop\%20Projects/Shop\%20Tricks/... You can...

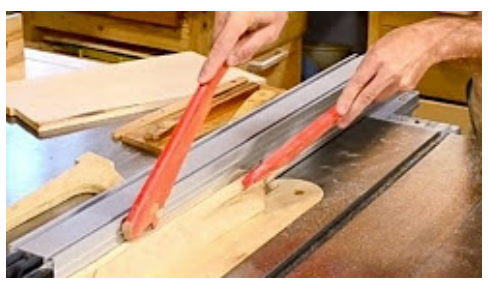

\section{Making push sticks}

Matthias Wandel

5 years ago $\cdot 320,228$ views

Showing off my favourite style of push sticks, and how to copy a shape

using my BigPrint program. http://woodgears.ca/table_saw/

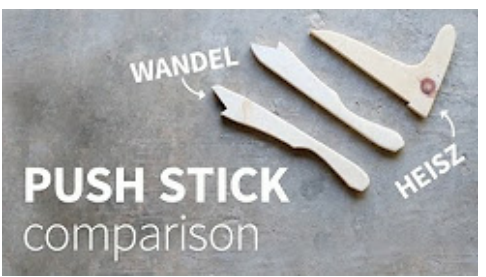

Wandel vs Heisz push stick comparison

EvanAndKatelyn

10 months ago $\bullet 16,869$ views

materials \& links below! **disclaimer** when we made this video, we were

still learning how to safely use our table saw and we did

Abb. 1.: Screenshot zu einem gemeinsamen Thema. https://www.youtube.com/results?search_ query=push-stick.

Darüber hinaus greifen die Video-Macherinnen und -Macher Kommentare auf, die zu ihren Videos erstellt wurden. Ein Beispiel dafür ist die Serie «John reads comment» von John Heisz (z.B. https://www.youtube.com/watch?v=Zrqg4CWzIMY). Auch im Stil der Videos ist erkennbar, dass sich die YouTuber in technischer und pädagogischer Hinsicht durch das Lernen voneinander und durch Kommentare professionalisieren: in Mode ist derzeit z.B. der Einsatz von Zeitraffern. Anregungen zum Lernen voneinander entstehen schliesslich durch sog. Challenges. Hier wird die YouTube-Community aufgefordert, innovative Lösungen zu entwickeln. Ein Beispiel ist die Challenge «no lathe pen», bei der ein Schreibstift ohne Einsatz einer Drehbank hergestellt 
werden sollte (https://www.youtube.com/playlist?list=PLHoCZ7LR6hNrJHIlhZxW876 wEhs9ReQTt). Durch solche Challenges entstehen offene Bildungsinhalte auch mit innovativem Charakter, die unterschiedliche Gewerke und Personen mit verschiedenen Erfahrungszusammenhängen untereinander vernetzen. YouTuber, die bisher eher passiv waren, werden dadurch angeregt, aktiv Inhalte einzustellen - auch, weil sie dadurch die Aufmerksamkeit auf sich lenken und Follower gewinnen können; erfahrene Macherinnen und Macher profitieren von kreativen Ideen der Novizen.

Interessant ist, dass auch die YouTuber nicht nur digitale Lernräume einnehmen, sondern auch klassische Werkstätten bzw. Maker Spaces bespielen oder aufbauen. Das informelle Lernen wird dann in einen formaleren Zusammenhang gebracht, z.B. werden Kurse zum Schmieden, zur Stahlbearbeitung, zu klassischen Holzverbindungen und zur Bedienung von CNC-Fräsen angeboten.

Das Beispiel der YouTuber verdeutlicht, dass die Plattform zum Knowledge Building (s.u.) anregt: die Video-Macherinnen und Macher lernen voneinander wie auch durch Kommentare, entwickeln mit der Zeit ihren eigenen Stil und stellen mit ihren auch innovativen Ideen «creative commons of practice» für alle zur Verfügung. Als Vernetzung und die Entwicklung einer kritisch-konstruktiven Haltung kann in diesem Beispiel die inhaltliche und stilistische Bezugnahme der YouTuber aufeinander und auf die Kommentierenden interpretiert werden.

\section{Berufsbezogenes Lernen mit digitalen Medien als situiertes Knowledge Building}

Wie können die zwei oben genannten Beispiele zum berufsbezogenen und gegenseitigen Lernen theoretisch gefasst werden? Einen wichtigen Ansatz zum Verstehen auch berufsbezogenen Lernens bieten Theorien situierter Kognition (Lave/Wenger 1999, Resnick et al. 1997, Scardamalia/Bereiter 2014). Die Grundidee dieses Ansatzes besteht darin, Denken und Sprache als unmittelbar miteinander verschränkt zu betrachten: «discourse is cognition is discourse» (Resnick et al. 1997, 2). Auch das Lernen erfolgt in diesem Sinne oft in Interaktion mit Menschen oder mit von ihnen geprägten Artefakten; in Praxisgemeinschaften, die durch bestimmte Regeln und Machtverteilungen geprägt sind (Lave/Wenger 1999, Fox 2000).

Theorien situierten Lernens sind eng mit Praxistheorien verwandt. Schäfer (2013) argumentiert:

«Grundsätzlich lässt sich jeder Ansatz als Praxistheorie begreifen, in dem Praktiken die fundamentale theoretische Kategorie oder den Ausgangspunkt einer empirischen Analyse bilden und damit eine Reihe etablierter philosophischer oder soziologischer Dichotomien zu überwinden sucht: etwa die Differenz zwischen Struktur und Handlung, Subjekt und Objekt, einer Regel und ihrer Anwendung, der Mikro- und Makroperspektive sowie zwischen Gesellschaft und Individuum» (ebd., 18). 
Scardamalia und Bereiter (2014) leiten aus der Theorie situierten Lernens sowie aus konnektivistischen und komplexittätstheoretischen Annahmen über selbstorganisierende Systeme Anforderungen an Lernumgebungen ab. In ihren Arbeiten fokussieren sie dabei auf schulische Umgebungen; ebenso lassen sich ihre Überlegungen aufgrund des theoretischen Hintergrundes auch auf die Erwachsenenbildung übertragen. Sie plädieren dafür, Lernumgebungen zu schaffen, das selbstorganisierte Lernen und den Aufbau von Wissen in einer Gemeinschaft, das sog. Knowledge Building zu unterstützen. Die Lernenden sollen Probleme durchdenken, fundiert erklären und reflektieren, neu definieren und in Handeln umsetzen (ebd., 7 ff.). Pädagogische Umgebungen, die ein solches Lernen fördern, beinhalten laut Scardamalia und Bereiter (2014)

- die Unterstützung der Setzung eigener Prioritäten, Selbstreflexion in Bezug auf die Zielerreichung und Förderung von Problemlösefähigkeiten;

- das Teilen von Verantwortung für und die Demokratisierung des Aufbaus von gemeinsamen Wissensbeständen;

- die Suche nach und Priorisierung vielversprechender Ideen;

- die Unterstützung von Diskursen, die Erklärungen liefern;

- die Loslösung von Ideen und Personen zur kritischen Weiterbearbeitung der Ideen;

- das Fördern von Risikobereitschaft und Unsicherheitstoleranz im Umgang mit neuen Ideen (ebd., 3 f.).

Das Mitdenken einer Gemeinschaft von Lernenden, der handlungsorientierte Ansatz und die Anregung epistemologischer Neugierde sind auch ein wichtiger Bestandteil von Reformandragogik, wie sie z.B. Paolo Freire (2001) formulierte.

Das berufsbezogene Lernen mit Twitter und YouTube kann gut mit der Metapher des Netzwerks gefasst werden: Wie Lisas Beispiel zeigt, werden einerseits individuell Fragmente (Erklärungen, Fertigkeiten, Ansprechpartnerinnen und -partner, Werkzeuge) zusammengesucht und als Ressourcen für innovatives Verhalten genutzt. Andererseits entstehen - wie im Beispiel der YouTuber - Prozesse gemeinsamen Wissensaufbaus, die durch das Internet gestützt werden und nicht notwendigerweise in der Erwerbsarbeit situiert sind. Das Netzwerk des Lernens besteht dabei nicht nur aus Menschen, sondern auch aus Artefakten wie schon geteilten Videos, Blogs, Tweets etc., und nicht zuletzt aus der technischen Umgebung, die eine Eigendynamik anregt (z.B. den Drang, regelmässig Videos in guter Qualität zu posten, um die Abonnentinnen und Abonnenten zu behalten) (Fox 2000, Resnick 1997). Die Charakteristika einer Knowledge Building Community, auf die Scardamalia und Bereiter (2014, s.o.) verweisen, werden dabei realisiert, was durch die Verfügbarkeit von Zeit, Personen und inhaltlichen Ressourcen sowie die Verknüpfung dieser Personen und Ressourcen untereinander vereinfacht wird. Durch den informellen Kontext von Social Media 
wird eine Offenheit für interdisziplinäres Denken und Lernen durch Zufall gefördert, die im formalen erwachsenenpädagogischen Zusammenhang oft fehlt. Solche Prozesse treten am ehesten dann auf, wenn die Lernenden Freiräume für den Austausch haben. Als Beispiel für ein non-formelles Weiterbildungsformat, in dem Netzwerken und Knowledge Building gefördert wird, benennt die Interviewperson Lisa BarCamps und Konferenzen mit der Möglichkeit intensiven Austausches:

«Also jeder, der da hingeht, kann selber eine Session anbieten und wenn ich jetzt sage: mich interessiert dieses Thema oder jenes, dann suche ich mir halt eines aus. Die BarCamp Liste ist ja insgesamt gross, es gibt jedes Wochenende fünf verschiedene Veranstaltungen gleichzeitig zu unterschiedlichen Schwerpunkten. Und dann läuft ja nicht nur immer eine Session, sondern meistens fünf parallel. Also von daher: wie lerne ich? Einmal Twitter, ja, aber BarCamps sind für mich auch noch eine recht gute Form, solange es nicht zu sehr Spezialwissen ist. Also ich sag mal für Spezialwissen würde ich dann doch auf eine Konferenz gehen, wenn dann dort halt Leute ganz detailliert bestimmte Dinge referieren, und dann auch die Möglichkeit eines intensiven Austausches gibt, weil nur für einen Vortrag, da kann ich mir auch ein Video angucken.» (Z. 553 ff.)

Wenn Anbieter beruflicher Weiterbildung in formellen Zusammenhängen Knowledge Building und creative commons of practice fördern wollen, können sie ihre eigenen Lernumgebungen authentischer und smarter gestalten (s.o., Scardamalia und Bereiter 2014), sie können Zeiten für das Lernen im BarCamp-Format anbieten, sie können aber auch den goldenen Weg des Verlinkens gehen und auf thematisch einschlägige bestehende Communities verweisen und daran anknüpfen (Verschlagwortung, Hilfestellung, Zusammenarbeit und Kommentierung, um mit der richtigen Zielgruppe in Kontakt zu kommen). Auf struktureller Ebene ist dabei denkbar, das Lernen in solchen informellen Netzwerken soweit anzuerkennen, dass es z.B. im Rahmen vom Weiterbildungsverpflichtungen angerechnet wird. So sieht z.B. die BerufskraftfahrerQualifikations-Verordnung (BKrFQV) vor, dass Berufskraftfahrerinnen und -kraftfahrer alle 5 Jahre 35 Stunden Weiterbildung nachweisen müssen. Diese Weiterbildung muss bislang in formellen analogen Sitzungen absolviert werden, die aufgrund ihres Formates und ihrer Standardisierung nicht für alle Teilnehmenden fruchtbar sind (vgl. Ball 2013).

\section{Medienkompetenzförderung von Fachkräften weit fassen}

Dem Internet wie auch der Erwachsenenbildung wird Ubiquität zugeschrieben (Jörissen 2013, Kade/Seitter 1996). Die Idee von Medienkompetenz schwankt - ebenso wie das Paradigma Lebenslangen Lernens - zwischen neoliberalem und reformpädagogischen Impetus; beide erfordern selbstorganisierte Lernprozesse. Aus Sicht der 
Medienpädagogik beanstandet Jörissen (2013), dass Förderangebote für Erwachsene entweder Mediengestaltung oder -kritik fokussierten, aber selten beides, und konstatiert fehlenden reflexiven Bezug zur mediatisierten Welt. Aus berufspädagogischer Sicht können wir dieser Kritik hinzufügen, dass Medienkompetenz oft entweder nur für allgemeine gesellschaftliche Teilhabe oder ausschliesslich mit Blick auf berufliche Handlungsfähigkeit gedacht und gefördert wird. Medienkompetenz gehört heute selbstverständlich zu beruflicher Handlungskompetenz: sei es, dass sie Teil beruflicher Aufgaben ist (z.B. in der Informatik), sei es, dass sie kompensatorisch zur Problemlösung benötigt wird, z.B. in Berufen, wo Fachkräfte oft allein und mit hoher Verantwortung arbeiten. Und doch sollte berufsbezogene Medienkompetenz auch als Befähigung zur Teilhabe in einer zunehmend digitalisierten Gesellschaft gedacht werden und als eine Form lebenslangen Lernens - wozu vor allem gehört, neugierig, kritisch und offen für neue Entwicklungen zu bleiben. Ein Ausschnitt einer konkreten Agenda zur Medienkompetenzförderung (MK) in der Logistik kann z.B. so aussehen:
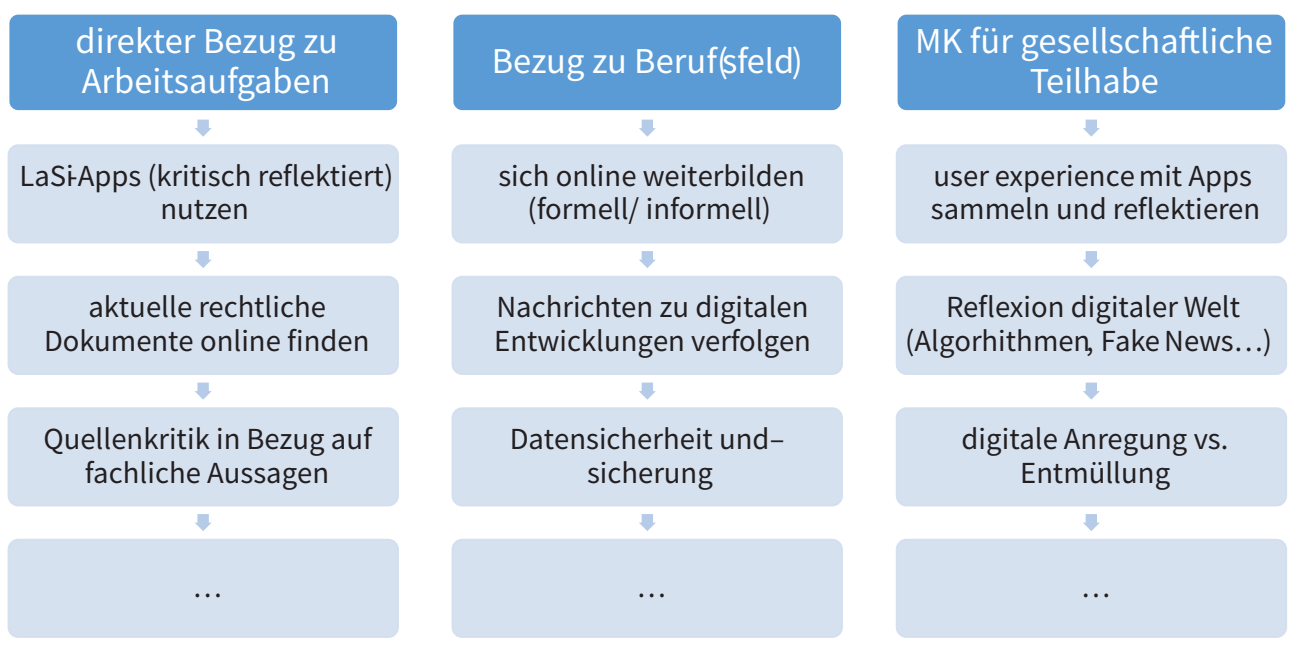

Abb. 2.: Grundlage für ein Medienkompetenz-Raster für die berufliche (Weiter-)Bildung.

Die Auseinandersetzung mit digitalen Medien, die im Arbeitsalltag benötigt werden - z.B. Apps zur Ladungssicherung - wird hier um den breiten Bezug zum Berufsfeld und die Förderung allgemeiner Medienkompetenz ergänzt. Diese allgemeine Medienkompetenz kann z.B. im Ausbau von Erfahrungen mit digitalen Medien bestehen - einem lustvollen, kritisch-reflexiven Probieren ohne unmittelbare Verwertungsnot. Die Erfahrung, die aus einer solchen allgemeinen Auseinandersetzung mit der mediatisierten Gesellschaft entsteht, erleichtert wiederum Engagement in Zusammenhängen, in denen fachliches Wissen sich zum gesellschaftlich geteilten Knowledge Building, zu creative commons of practice weiterentwickelt. Zu einer digitalisierten Gesellschaft gehört auch, dass disziplinäre Grenzen überschritten und die Sicherheit tradierter Formen der Wissensweitergabe gebrochen wird. Durch Social Media 
wie YouTube und Twitter erfahren Millionen von Menschen bereits neue Formen des Lernens und Lehrens, die nicht nur methodisch neue Wege beschreiten, sondern auch inhaltlich zu innovativen Auseinandersetzungen, z.B. mit Materialkunde, Entstehungs- und Entsorgungsprozessen und damit zu alternativen Handlungsweisen und Antworten auf globale Herausforderungen führen.

Die Absicht unseres Artikels besteht nicht darin, Social Media als ideale Lernumgebung darzustellen. Wir wissen, dass ökonomische Verwertungsinteressen die Technikgestaltung mitprägen, dass Algorithmen mitbestimmen, wer was sieht; dass soziale Interaktion im Internet genauso fehleranfällig ist wie offline (Was wird gefiltert? Wer wird gemobbt? Was wird ignoriert, obwohl es gut ist?) und dass das Transferproblem beruflicher Weiterbildung nicht einfach dadurch gelöst wird, dass man mit Social Media lernt (Köster 2003). Wir wissen, dass zu digitaler Mündigkeit auch digitale Müdigkeit gehört. Aber wir wollen doch explizit auf die Chancen digitaler Lernräume hinweisen und darauf, dass digitalisiertes Lernen mehr ist als die Nutzung von Ressourcen im Internet. Durch Vernetzung in digitalen Räumen entsteht etwas Anderes als die Summe seiner Teile - eine amorphe Lernumgebung, die gerade das berufliche Lernen von Expertinnen und Experten bereichern kann. Die Schranken zwischen Lernenden und Lehrenden können hier aufgehoben werden und Raum für demokratische Teilhabe schaffen, die nicht digital bleibt.

\section{Literatur}

Ball, Claudia. 2013. «Berufliche Aus- und Weiterbildung und Lebenslanges Lernen von Berufskraftfahrern: Chancen und Risiken einer europaweiten Pflicht zur regelmässigen Weiterbildung».bwp@ Spezial 6 - Hochschultage Berufliche Bildung, 1-19. http://www.bwpat.de/ ht2013/ft08/ball_ft08-ht2013.pdf.

Bundesverband betriebliche Weiterbildung (2016). Trendstudie des Wuppertaler Kreis e.V. http://www.wkr-ev.de.

Burchert, Joanna. 2014. Von der Facharbeit in die Ausbildung: Empirische Studie und theoretischer Rahmen zur Erforschung des pädagogischen Selbstverständnisses hauptamtlicher Ausbilder und Ausbilderinnen. Dissertation an der Universität Bremen. http://elib.suub.unibremen.de/edocs/00104110-1.pdf.

Burchert, Joanna, und Rasmus Grobe. 2017. «Herausforderungen bei der Implementierung digital gestützter beruflicher Weiterbildung. Die Sicht von WeiterbildnerInnen und BildungsmanagerInnen auf Strukturen, kulturelle Praktiken und Agency». Magazin erwachsenenbildung.at Das Fachmedium für Forschung, Praxis und Diskurs, 30. http://www.erwachsenenbildung.at/magazin/17-30/meb17-30.pdf.

Fenwick, Tara, und Richard Edwards. 2010. Actor-Network Theory in Education. London: Routledge. 
Freire, Paolo. 2001. Pedagogy of Freedom: Ethics, Democracy, and Civic Courage. Lanham: Rowman and Littlefeld.

Fox, Stephen. 2000. «Communities Of Practice, Foucault And Actor-Network Theory». Journal of Management Studies 37- 6, 853-868. http://onlinelibrary.wiley.com/doi/10.1111/1467$6486.00207 /$ full.

Hattie, John. 2009. Visible learning. A synthesis of over 800 meta-analyses relating to achievement. London, New York: Routledge.

Hart, Jane. 2016. Top Tools for Learning 2016. http://c4lpt.co.uk/top100tools/top-200-toolsfor-learning/.

Jörissen, Benjamin. 2013. «Unbestellte Bildungsfelder - wo bleiben die neuen Formate der Erwachsenen- und Weiterbildung?» Forum Erwachsenenbildung, Nr. 2: 16-21.

Kade, Jochen, und Wolfgang Seitter. 1996. Lebenslanges Lernen - mögliche Bildungswelten: Erwachsenenbildung, Biographie und Alltag. Opladen: Leske + Budrich.

Köster, M. 2003. «Warum Training selten funktioniert: Über die Notwendigkeit von soziologischer Perspektive in einer boomenden Branche». Sozialwissenschaften und Berufspraxis (SuB) 26, 255-267.

Larbig, Torsten, und André Spang, Hrsg. 2017. Digitale Medien für Unterricht, Lehrerjob und Schule: die besten Ideen und Tipps aus dem Twitterchat \#EDchatDE. 1. Auflage. Berlin: Cornelsen. https://news.rpi-virtuell.de/wp-content/uploads/2017/02/digitale-medien-furunterricht.pdf.

Lave, Jean, und Etienne Wenger. 1999. Situated learning. Legitimate peripheral participation. Cambridge.

Podrath, Gudrun.2016.Lernen mit Youtube. https://www.haufe.de/personal/hr-management/elearning-lernen-mit-youtube_80_380714.html

Resnick, Lauren B., Clothilde Pontecorvo, und Roger Saljö. 1997. «Discourse, tools and reasoning». In Discourse, tools and reasoning: Essays on situated cognition, hrsg. von Lauren B. Resnick, Roger Saljö, Clothilde Pontecorvo und Barbara Burge, 1-22. Berlin u.a.: Springer.

Scardamalia, Marlene, und Carl Bereiter. 2014. «Smart technology for self-organizing processes». Smart Learning Environments 1:1, http://slejournal.com/content/1/1/1.

von Rein, Nuissl im Interview mit Ion Dumitru. 2000. Erwachsene lernen anders. http://www. die-frankfurt.de/esprid/dokumente/doc-2000/dumitru00_01.htm.

Wolf, Gertrud. 2016. «Learning by YouTube. Was macht die Video-Plattform als informelles Lernangebot so erfolgreich?». Erwachsenenbildung 62, Heft 2, 62-64. 